\title{
Pinned magnetic moments in exchange bias: Role of the antiferromagnetic bulk spin structure
}

\author{
M. Yaqoob Khan, ${ }^{*}$ Chii-Bin Wu (吳啟涁) ${ }^{\dagger}$ and Wolfgang Kuch ${ }^{\ddagger}$ \\ Institut für Experimentalphysik, Freie Universität Berlin, Arnimallee 14, 14195 Berlin, Germany \\ (Received 1 November 2013; revised manuscript received 14 March 2014; published 31 March 2014)
}

\begin{abstract}
Magneto-optical Kerr effect measurements of epitaxial AFM/FM bilayers and FM/AFM/FM trilayers on $\mathrm{Cu}_{3} \mathrm{Au}(001)$, where "AFM" stands for a $\mathrm{Ni}_{25} \mathrm{Mn}_{75}$ antiferromagnetic layer, and "FM" for ferromagnetic layers that are either $\mathrm{Ni}$ or $\mathrm{Ni} / \mathrm{Co}$ with out-of-plane or in-plane easy axis of magnetization, show that trilayers with collinear magnetization directions of both FM layers exhibit always a much lower exchange bias field $H_{\mathrm{eb}}$ at a fixed temperature compared to bilayers of the same $\mathrm{Ni}_{25} \mathrm{Mn}_{75}$ thickness. At the same time, the blocking temperature for exchange bias $T_{b}$ is strongly reduced. In trilayers with orthogonal easy axes of the two FM layers (in-plane and out-of-plane), in contrast, both $H_{\mathrm{eb}}$ and $T_{b}$ are nearly identical to that of the corresponding bilayers. Such a behavior can be explained by pinned magnetic moments inside the bulk of the AFM layer that coexist independently for orthogonal spin directions, but have to be equally shared between both interfaces in the case of collinear spin directions. This result thus also confirms a $3 Q$-like noncollinear spin structure of $\mathrm{Ni}_{25} \mathrm{Mn}_{75}$.
\end{abstract}

DOI: 10.1103/PhysRevB.89.094427

PACS number(s): 75.70.Ak, 75.30.Et, 75.50.Ee, 75.70.Cn

\section{INTRODUCTION}

The unidirectional anisotropy in the exchange bias (EB) effect [1-3] is caused by the simultaneous presence of two essential ingredients: exchange coupling at the interface between a ferromagnetic (FM) and an antiferromagnetic (AFM) material and the presence of pinned magnetic moments after the sample has been cooled in a magnetic field. Due to its technological relevance and interesting physics, a lot of effort has been dedicated to reveal the detailed nature of the underlying mechanism [2-23]. In an early model by the discoverers of the EB effect, the entire spin structure of the AFM layer has been assumed to be pinned, while all the interfacial AFM spins contributed to the exchange coupling with the FM layer [4]. Later on, refined models have been put forward to improve the quantitative agreement with experimental observations [5-10]. The AFM-FM coupling is mediated by uncompensated moments of the AFM layer at the interface, which either result from the intrinsic AFM spin structure or may be induced, for example, at step edges, by the presence of the FM layer [11-14]. An AFM-FM magnetic coupling, which usually manifests itself by an increased coercivity [15], alone does not yet lead to exchange bias. In addition, also some spins of the system need to be pinned in a certain direction after the field cooling to define the direction of the exchange bias field $H_{\mathrm{eb}}$, the horizontal shift of the magnetization loop along the field axis. Finally, the pinned moments need to couple to the FM layer and/or the uncompensated rotatable moments responsible for the AFM-FM coupling.

The nature of the pinned moments is still controversial, but several models agree in that some kind of disorder is necessary [7-9]. The location of these pinned moments is under debate, too. Experimentally, pinned uncompensated moments have

\footnotetext{
*Present address: Kohat University of Science and Technology, Kohat 26000, Khyber Pukhtunkhwa, Pakistan.

${ }^{\dagger}$ Present address: Chung Yuan Christian University, 200 Chung Pei Rd., Chung Li City, Taiwan 32023, R.O.C.

${ }^{\ddagger}$ kuch@physik.fu-berlin.de
}

been identified in some EB systems, and assumed to be located at the interface [16], such that they would couple directly to the FM layer. Other experiments, however, support a distribution of the pinned moments over the entire bulk of the AFM layer $[17,18]$. In that case, the question is in which direction these uncompensated moments point and how they interact with the FM layer through the AFM material.

Studying FM/AFM/FM trilayers allows to obtain information about the AFM layer that can not be accessed easily from experiments on FM/AFM bilayers. Detailed knowledge about the dependence of the AFM-FM magnetic interface coupling on the atomic scale interface roughness has been gained from trilayer experiments [12]. Yang and Chien reported an independent EB effect in the two FM layers of a permalloy $(\mathrm{Py}) / \mathrm{FeMn} / \mathrm{Co}$ trilayer as long as the FeMn AFM layer thickness was larger than $9 \mathrm{~nm}$ [19]. Morales et al., in contrast, observed in a $\mathrm{Ni} / \mathrm{FeF}_{2} / \mathrm{Py}$ trilayer with $200-\mathrm{nm}$ AFM layer thickness that $H_{\mathrm{eb}}$ was smaller when the two FM layers had antiparallel EB compared to the case of parallel EB [18]. Blamire et al. found that the presence of a Co layer in $\mathrm{Co} / \mathrm{FeMn} / \mathrm{CuNi}$ trilayers had an influence on the $\mathrm{EB}$ of the $\mathrm{CuNi}$ layer only for FeMn thicknesses below about $2 \mathrm{~nm}$ [10].

Here, we present experimental results on epitaxial $\mathrm{FM} / \mathrm{Ni}_{25} \mathrm{Mn}_{75} / \mathrm{FM}$ trilayers on $\mathrm{Cu}_{3} \mathrm{Au}(001)$. The $\mathrm{FM}$ layers either consist of $\mathrm{Ni}$, which has an out-of-plane (OoP) easy axis of magnetization on $\mathrm{Cu}_{3} \mathrm{Au}(001)$ [24], or of $\mathrm{Co} / \mathrm{Ni}$. By adding a thin Co underlayer (overlayer) to the bottom (top) Ni FM layer, the easy axis of magnetization of the combined $\mathrm{Ni} / \mathrm{Co}$ layer is manipulated to be in-plane (IP), while still having the same $\mathrm{Ni} / \mathrm{Ni}_{25} \mathrm{Mn}_{75}$ interface. Single-crystalline systems have the advantage over polycrystalline or sputtered systems that structural properties, in particular at the AFM-FM interface, can be better controlled [12]. We have shown previously that the surface roughness and crystalline structure of a $\mathrm{Ni}$ film grown on top of a thin Co layer on $\mathrm{Cu}_{3} \mathrm{Au}(001)$ is very similar to the one of Ni directly deposited on $\mathrm{Cu}_{3} \mathrm{Au}(001)$ [20]. $\mathrm{Ni}_{x} \mathrm{Mn}_{100-x} \mathrm{AFM}$ layers grown on top of $\mathrm{Ni} / \mathrm{Cu}_{3} \mathrm{Au}(001)$ or $\mathrm{Ni} / \mathrm{Co} / \mathrm{Cu}_{3} \mathrm{Au}(001)$ couple to the FM layer both for IP and OoP easy axis of magnetization in a wide range of concentrations $x$, while a lower $x$ leads to a higher blocking temperature for EB [21]. 


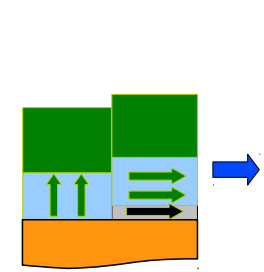

(a)

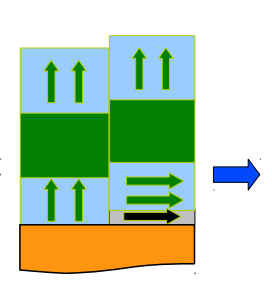

(b)

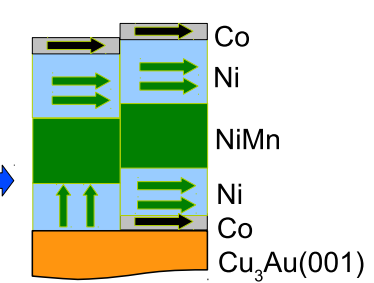

(c)
FIG. 1. (Color online) Schematic illustration of the idea of the experiment. By depositing a thin $(\approx 2 \mathrm{ML})$ Co underlayer on one half of the substrate before evaporation of the $\mathrm{Ni} \mathrm{FM}$ and $\mathrm{Ni}_{25} \mathrm{Mn}_{75} \mathrm{AFM}$ layers, successively, the following configurations can be measured: OoP and IP bilayers (a), collinear OoP-OoP and noncollinear IPOoP trilayers after the deposition of the top Ni FM layer (b), and noncollinear OoP-IP and collinear IP-IP trilayers after deposition of a thin $(\approx 3 \mathrm{ML})$ Co overlayer (c).

Figure 1 illustrates the scheme of our experiment. Samples were grown and measured in three steps. At first, a thin layer of Co is deposited on only one half of the substrate with the help of a mechanical shutter. The subsequently deposited $\mathrm{Ni}$ layer then displays an OoP easy axis of magnetization on bare $\mathrm{Cu}_{3} \mathrm{Au}(001)$ [24], but IP magnetization on top of the Co layer due to the additional anisotropy of the Co layer. After deposition of a $\mathrm{Ni}_{25} \mathrm{Mn}_{75}$ layer [Fig. 1(a)], the exchange bias field and the coercivity of the sample is measured as a function of temperature by longitudinal and polar MOKE in the two halves of the sample. The second step consists of depositing a top Ni FM layer, which again displays OoP anisotropy [Fig. 1(b)]. The sample now has a collinear OoPOoP configuration in one half and noncollinear IP-OoP in the other. Finally, a thin Co layer deposited on top changes the magnetic easy axis of the top FM layer to IP [Fig. 1(c)]. Now the sample is noncollinear OoP-IP on one side, and collinear IP-IP on the other. We find that the EB field of the bottom FM layer decreases dramatically upon the presence of a top FM layer with identical easy axis of magnetization, while it is nearly insensitive to the presence of a top FM layer with a different easy axis of magnetization. This can be interpreted in terms of pinned magnetic moments distributed inside the bulk of the AFM layer that can coexist independently for orthogonal spin directions but have to be equally shared between both interfaces in the case of collinear spin directions.

Our results agree with the findings of Morales et al. [18] in that the pinned uncompensated AFM moments that give rise to EB are located not only at the interface, but throughout the entire bulk AFM magnetic structure. Here, we compare the EB in trilayers with parallel EB with that in a bilayer, using the very same AFM layer. We show that the pinned moments and the FM layers interact in a nontrivial way such that pinning centers, in general, do not contribute simultaneously to the EB of collinearly magnetized FM layers at both sides of the AFM layer, even though they interact with both layers. Finally, we demonstrate that different pinning centers exist that primarily lead to EB along either the IP or the OoP magnetization direction, supporting the assumption of a noncollinear spin structure in the AFM layer.

\section{EXPERIMENT}

The experiments were performed under ultrahigh vacuum (UHV) conditions with a base pressure of about $10^{-10}$ mbar. The single-crystalline $\mathrm{Cu}_{3} \mathrm{Au}(001)$ substrate was cleaned by sputtering with $1 \mathrm{keV} \mathrm{Ar}^{+}$ions and annealing at $800 \mathrm{~K}$ for 10 minutes. All the three materials were deposited from high-purity (Co and Ni: 99.99\%, Mn: 99.95\%) rods by electron bombardment with the substrate held at room temperature. Film thicknesses were calibrated by medium energy electron diffraction and Auger electron spectroscopy, as described elsewhere [21]. Approximately two atomic monolayers (ML) Co were deposited on one half of the $\mathrm{Cu}_{3} \mathrm{Au}(001)$ substrate by the use of a mechanical shutter. Subsequently, a Ni layer of $12 \mathrm{ML}$ thickness was deposited over the entire sample, followed by a $\mathrm{Ni}_{25} \mathrm{Mn}_{75}$ layer of $27 \mathrm{ML}$ thickness. The thickness of the top $\mathrm{Ni}$ layer was $22 \mathrm{ML}$. $\mathrm{Ni}_{25} \mathrm{Mn}_{75}$ was grown by coevaporation of $\mathrm{Ni}$ and $\mathrm{Mn}$ from separate sources. The accuracy of the thickness determination is $\pm 1 \mathrm{ML}$ for $\mathrm{Ni}_{25} \mathrm{Mn}_{75}$ and sub-ML for $\mathrm{Ni}$ and $\mathrm{Co}$, the error in the $\mathrm{Ni}(\mathrm{Mn})$ concentration is less than $\pm 2 \%$.

Before each measurement series, the samples were fieldcooled from $490 \mathrm{~K}$ in a field of $+20 \mathrm{mT}$ along the easy axis of the respective probed interface. Magnetization loops are subsequently recorded using MOKE, while increasing the temperature from lower to higher values. Linearly polarized laser light of $1-\mathrm{mW}$ power and $635-\mathrm{nm}$ wavelength was used for the in situ MOKE measurements. For these measurements, the sample was placed in a glass finger sitting in between the two poles of an electromagnet. A straight line was subtracted from the resulting magnetization curves to account for the Faraday effect of the glass finger.

\section{RESULTS AND DISCUSSION}

Three representative magnetization loops measured by polar MOKE at $300 \mathrm{~K}$ for the OoP bilayer (top curve), OoP-OoP (middle curve), and OoP-IP trilayer (bottom curve) are shown in Fig. 2. In the OoP-OoP case, the separate switching of the top and bottom FM layers is clearly observed, where the smaller step at higher field can be attributed to the bottom layer because of its smaller thickness and larger signal damping. The other two loops represent only the bottom layer. It is evident that $H_{\mathrm{eb}}$ present in the bilayer is completely vanishing (from -15 to $0 \mathrm{mT}$ ) when the second OoP FM layer is present on top of the OoP bilayer. This decrease in $H_{\mathrm{eb}}$ is also observed in the IP-IP trilayer of the same sample as well as in IP-IP and OoP-OoP trilayers with a larger $\mathrm{Ni}_{25} \mathrm{Mn}_{75}$ thickness measured in the same way [25]. After the top FM layer is made IP by depositing $\mathrm{Co}, H_{\mathrm{eb}}$ of the OoP-IP trilayer now once again increases (from zero to $-7 \mathrm{mT}$ ) as can be seen from the bottom loop of Fig. 2 .

The resulting $H_{C}(T)$ and $H_{\mathrm{eb}}(T)$ of the bottom layer during the three steps are shown in Figs. 3(a) and 3(b) for IP and OoP magnetization, respectively. Interestingly, in both cases, the EB at a fixed temperature is lowest in the collinear case (blue triangles). For IP bottom layer [Fig. 3(a)], the EB field as well as the coercivity of the bilayer (green squares) are rather unchanged after the deposition of the OoP top layer (red circles), but change markedly just by turning the magnetization 


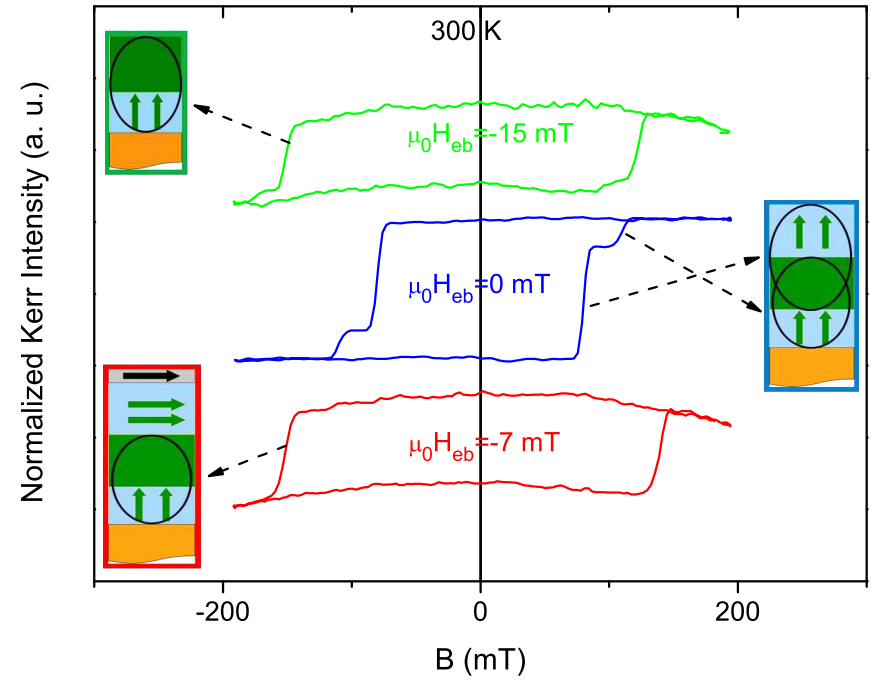

FIG. 2. (Color online) Normalized hysteresis loops measured by polar MOKE at $300 \mathrm{~K}$. OoP bilayer $27 \mathrm{ML} \mathrm{Ni}_{25} \mathrm{Mn}_{75} / 12 \mathrm{ML}$ $\mathrm{Ni} / \mathrm{Cu}_{3} \mathrm{Au}$ (001) (top loop), OoP-OoP trilayer $22 \mathrm{ML} \mathrm{Ni} / 27 \mathrm{ML}$ $\mathrm{Ni}_{25} \mathrm{Mn}_{75} / 12 \mathrm{ML} \mathrm{Ni} / \mathrm{Cu}_{3} \mathrm{Au}(001)$ (middle loop), and OoP-IP trilayer (bottom loop). All measurements are performed after $\mathrm{FC}$ with $+20 \mathrm{mT}$ perpendicular to the interface from $490 \mathrm{~K}$. The OoP-OoP trilayer (middle loop) completely vanishes the EB field of the OoP bilayer. Due to the large coercivity, one should be careful in observing the top and bottom loop shift to the negative of the field axis. The easy axis of magnetization of the FM layers and the possible spin configuration of the AFM layer near the interface are also schematically shown for each loop.

of the top layer into the IP direction (blue triangles). The $H_{\mathrm{eb}}(T)$ and $T_{b}$ are smallest for the IP-IP trilayer compared to the IP bilayer and the IP-OoP trilayers. The blocking temperature for EB, $T_{b}$, is $\approx 150 \mathrm{~K}$ smaller in the IP-IP trilayer compared to the IP bilayer or the IP-OoP trilayer. A peak in $H_{C}(T)$ at around the blocking temperature is observed in all three curves.

A small positive EB just below $T_{b}$ can be noted for the IP bottom FM layer in all the three cases [25]. This is consistent with the results obtained for a $\mathrm{Ni}_{81} \mathrm{Fe}_{19} / \mathrm{Ir}_{20} \mathrm{Mn}_{80}$ bilayer (Ref. [23]), and can be attributed to an unusual minority species of pinned spins, which strongly pin the FM layer in opposite direction to that of the majority pinned spins.

The situation is similar for the OoP bottom layer [Fig. 3(b)]. Here, the collinear trilayer (OoP-OoP) is achieved in the second step (blue triangles). Again, its EB field and $T_{b}$ are much lower than in the bilayer. By turning the top layer magnetization into the plane, the noncollinear OoP-IP configuration is achieved (red circles), and $H_{\mathrm{EB}}$ at fixed temperature as well as $T_{b}$ of the bottom layer clearly increase, albeit not fully reaching the values of the bilayer. In a certain temperature window around $320 \mathrm{~K}$, the $\mathrm{EB}$ is switched on just by spin engineering the top FM layer magnetization from OoP to IP. A similar, but weaker effect is also found for $41 \mathrm{ML}$ $\mathrm{Ni}_{25} \mathrm{Mn}_{75}$ thickness [25].

We explain our findings by the following model: As in the domain-state model $[8,17]$, we assume pinning centers within the volume of the AFM layer. These could result from the chemical disorder in the $\mathrm{Ni}_{25} \mathrm{Mn}_{75}$ alloy. They consist of
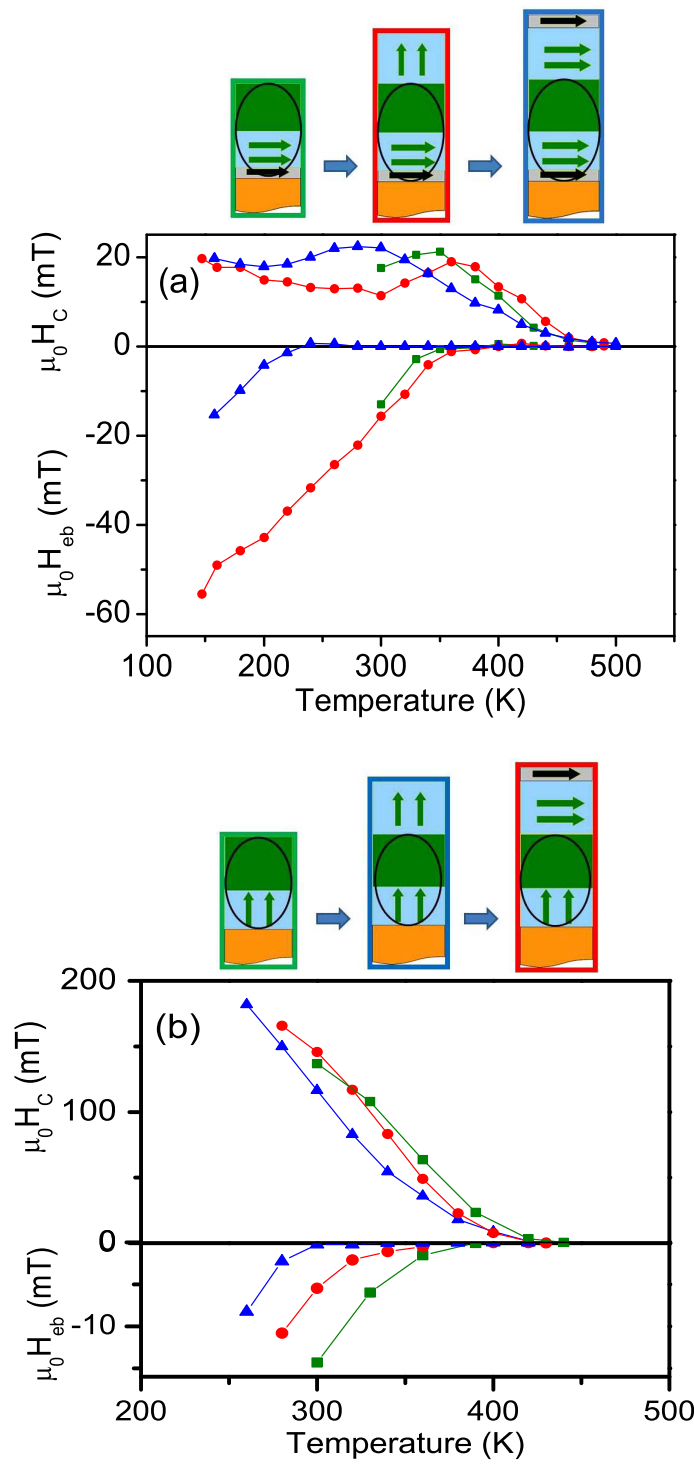

FIG. 3. (Color online) Temperature dependence of coercivity $H_{C}$ and exchange bias field $H_{\mathrm{eb}}$ of the $27 \mathrm{ML} \mathrm{Ni}_{25} \mathrm{Mn}_{75} / 12 \mathrm{ML} \mathrm{Ni} /(2 \mathrm{ML}$ $\mathrm{Co} /) \mathrm{Cu}_{3} \mathrm{Au}(001)$ bilayer (squares) and for the bottom layer of a (3 ML $\mathrm{Co} /) 22 \mathrm{ML} \mathrm{Ni} / 27 \mathrm{ML} \mathrm{Ni}_{25} \mathrm{Mn}_{75} / 12 \mathrm{ML} \mathrm{Ni} /(2 \mathrm{ML} \mathrm{Co} /) \mathrm{Cu}_{3} \mathrm{Au}(001)$ trilayer in the noncollinear (circles) and the collinear configuration (triangles) measured with (a) longitudinal and (b) polar MOKE after field cooling each sample in a 20-mT field along the easy axis of the bottom layer from $490 \mathrm{~K}$. The magnetization directions of the FM layers at the upper and at the lower interfaces are schematically shown on top of the respective graph, where ellipses indicate the measured FM layer. Light blue represents $\mathrm{Ni}$, green $\mathrm{Ni}_{25} \mathrm{Mn}_{75}$, and grey $\mathrm{Co}$ layers. The color of the frame of the schematics corresponds to the color of the $H_{C}(\mathrm{~T})$ and $H_{\mathrm{eb}}(\mathrm{T})$ curves.

pinned uncompensated moments. The important point is that these pinned moments do not have to be aligned with the direction of the exchange bias field. From the small influence a top FM layer with different easy axis of magnetization has on the bottom layer, it follows that the pinning centers responsible for IP and OoP EB are to a large extent independent. The FM layers couple at the interface to the spin structure of the AFM layer. Without pinning centers, this just leads to an enhanced coercivity. The pinning centers couple to the FM layer via 
(a)

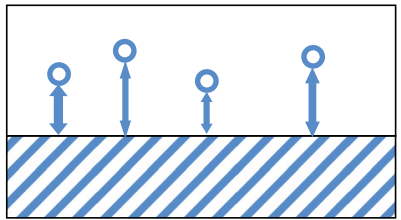

(b)

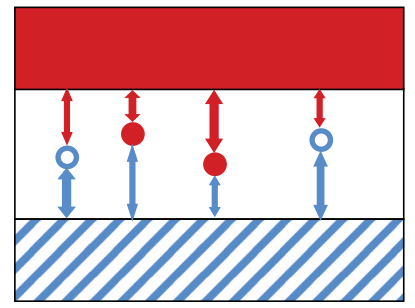

FIG. 4. (Color online) Sketch illustrating the proposed model of pinning centers in an AFM/FM bilayer (a) and in a collinear FM/AFM/FM trilayer (b). The thickness of the arrows indicates the strength of the coupling between the pinning centers and the FM layer. Open (filled) circles in (b) are pinning centers in the AFM layer that provide pinning for the bottom (top) FM layer after field cooling.

exchange coupling through all the AFM spins between the pinning center and the interface. The detailed nature of this exchange path determines the strength of the coupling and the relative direction between the FM layer magnetization and pinned uncompensated moment. In an AFM with noncollinear spin structure, the pinned uncompensated moment does not need to be coupled to the FM layer magnetization in a collinear or parallel way. Upon magnetization reversal of the FM layer, this exchange path acts like an exchange spring, leading to EB. The pinned uncompensated moments may point along any IP direction for IP FM layer magnetization, and along the two OoP directions for OoP FM layer magnetization. Figure 4(a) schematically illustrates such a pinning of a bottom FM layer by pinning centers, coupled to the FM layer by exchange paths of different strength, indicated by the thickness of the arrows. If now a second FM layer of the same easy axis is present at the other interface of the AFM layer, the two layers compete for the pinning centers, even when they are magnetized in parallel. During field cooling, the pinned moments will then align according to the direction defined by the stronger of the two exchange paths, and hence provide EB only for one of the two FM layers. This is illustrated in Fig. 4(b). Here some of the pinning centers after field cooling now provide EB to the top FM layer. This reduces the effective thickness of the AFM layer available for each of the two FM layers, and leads to the observed reduction in $H_{\mathrm{eb}}$ and $T_{b}$.

There might be also some pinning centers that pin a spin under an oblique direction, and act at the same time on an IP and an OoP FM layer. This would explain why there is still a small difference between the EB of the bottom layer in the bilayer and in the noncollinear trilayer, as seen from the difference between the green and red curves (squares and circles, respectively) in Fig. 3. In a thinner AFM layer, there are less pinning centers, but with on average shorter and consequently stronger exchange paths. Stronger coupling between a pinning center and the FM layer results in a higher torque on the pinned moment upon FM layer magnetization reversal, which could make it easier to break the pinning thermally. In other words, if a pinning center is too close to the FM layer, no spin spiral or local horizontal domain wall might be accommodated, which would lead either to domains in the FM layer [22] or to the unpinning of the uncompensated moments. Following that idea, at higher temperatures, more distant pinning centers would thus dominate the EB, which consequently could exist only at larger film thicknesses. This would explain the increase of $H_{\mathrm{eb}}$ and $T_{b}$ with AFM layer thickness.

Interlayer coupling between the two FM layers can be excluded as the cause for the reduced EB. The two layers still switch at distinctly different fields (see Fig. 2). In that case, if a sizable ferromagnetic coupling between the two FM layers were present, it would decrease the switching fields of the bottom layer in both directions identically and thus its coercivity, but would not alter the EB field.

After the top-layer magnetization is switched from OoP to IP, the OoP bottom layer regains a higher EB field [Fig. 3(b)]. This means that the spin orientation on the upper interface significantly affects the $H_{\mathrm{eb}}$ of the lower interface in this kind of trilayer system. In our model all the IP pinning centers are now made available for pinning the bottom layer. The slight reduction compared to the case of the bilayer may be attributed to some pinning centers with canted pinning direction, which are still pinning the top FM layer also in the IP direction. The effect is independent of the bottom layer easy axis of magnetization. This and the much smaller influence of a top FM layer of orthogonal magnetization direction point towards a noncollinear AFM spin structure, such as the $3 Q$ spin structure, which allows an independent setting of the EB along IP and OoP spin components.

\section{CONCLUSION}

We conclude that pinning centers in the bulk of the AFM spin structure are responsible for the emergence of EB. Largely independent pinning centers exist for the pinning of IP and OoP magnetization directions. The uncompensated moments associated with these pinning centers are not necessarily aligned along the EB field. In trilayers with collinear magnetization of the two FM layers, the individual pinning centers are acting only on one of the FM layers, thus reducing the effective AFM layer thickness for EB for each of the FM layers. This manifests itself in a shift of the $H_{C}(T)$ and $H_{\mathrm{eb}}(T)$ curves to lower temperatures, and a concomitant decrease of the blocking temperature by up to $\approx 150 \mathrm{~K}$. Besides the fundamental insight it provides, this effect could be also used to manipulate the EB field acting on an FM layer by just switching the magnetization direction of another FM layer between IP and OoP.

\section{ACKNOWLEDGMENTS}

M. Y. Khan is grateful for financial support during his stay in Berlin by the Higher Education Commission (HEC) of Pakistan through Kohat University of Science \& Technology (KUST), Kohat, Pakistan, and Freie Universität Berlin.
[1] W. H. Meiklejohn and C. P. Bean, Phys. Rev. 102, 1413 (1956).

[2] J. Nogués and I. K. Schuller, J. Magn. Magn. Mater. 192, 203 (1999).
[3] F. Radu and H. Zabel, in Magnetic Heterostructures, edited by H. Zabel and S. D. Bader (Springer, Berlin, Heidelberg, 2008).

[4] W. H. Meiklejohn and C. P. Bean, Phys. Rev. 105, 904 (1957). 
[5] D. Mauri, H. C. Siegmann, P. S. Bagus, and E. Kay, J. Appl. Phys. 62, 3047 (1987).

[6] N. C. Koon, Phys. Rev. Lett. 78, 4865 (1997).

[7] A. P. Malozemoff, Phys. Rev. B 35, 3679 (1987).

[8] U. Nowak, K. D. Usadel, J. Keller, P. Miltényi, B. Beschoten, and G. Güntherodt, Phys. Rev. B 66, 014430 (2002).

[9] M. Ali, P. Adie, C. H. Marrows, D. Greig, B. J. Hickey, and R. L. Stamps, Nat. Mater. 6, 70 (2007).

[10] M. G. Blamire, M. Ali, C.-W. Leung, C. H. Marrows, and B. J. Hickey, Phys. Rev. Lett. 98, 217202 (2007).

[11] F. Offi, W. Kuch, L. I. Chelaru, K. Fukumoto, M. Kotsugi, and J. Kirschner, Phys. Rev. B 67, 094419 (2003).

[12] W. Kuch, L. I. Chelaru, F. Offi, J. Wang, M. Kotsugi, and J. Kirschner, Nat. Mater. 5, 128 (2006).

[13] C. Won, Y. Z. Wu, H. W. Zhao, A. Scholl, A. Doran, W. Kim, T. L. Owens, X. F. Jin, and Z. Q. Qiu, Phys. Rev. B 71, 024406 (2005).

[14] J. Choi, J. Wu, Y. Z. Wu, C. Won, A. Scholl, A. Doran, T. Owens, and Z. Q. Qiu, Phys. Rev. B 76, 054407 (2007).

[15] M. D. Stiles and R. D. McMichael, Phys. Rev. B 63, 064405 (2001).
[16] H. Ohldag, A. Scholl, F. Nolting, E. Arenholz, S. Maat, A. T. Young, M. Carey, and J. Stöhr, Phys. Rev. Lett. 91, 017203 (2003).

[17] P. Miltényi, M. Gierlings, J. Keller, B. Beschoten, G. Güntherodt, U. Nowak, and K. D. Usadel, Phys. Rev. Lett. 84, 4224 (2000).

[18] R. Morales, Zhi-Pan Li, J. Olamit, Kai Liu, J. M. Alameda, and I. K. Schuller, Phys. Rev. Lett. 102, 097201 (2009).

[19] F. Y. Yang and C. L. Chien, Phys. Rev. Lett. 85, 2597 (2000).

[20] M. Y. Khan, C.-B. Wu, M. Erkovan, and W. Kuch, J. Appl. Phys. 113, 023913 (2013).

[21] M. Y. Khan, C.-B. Wu, S. K. Kreft, and W. Kuch, J. Phys.: Condens. Matter 25, 386005 (2013).

[22] W. Kuch, F. Offi, L. I. Chelaru, M. Kotsugi, K. Fukumoto, and J. Kirschner, Phys. Rev. B 65, 140408(R) (2002).

[23] S. K. Mishra, F. Radu, H. A. Dürr, and W. Eberhardt, Phys. Rev. Lett. 102, 177208 (2009).

[24] A. Braun, B. Feldmann, and M. Wuttig, J. Magn. Magn. Mater. 171, 16 (1997)

[25] See Supplemental Material at http://link.aps.org/supplemental/ 10.1103/PhysRevB.89.094427 for further experimental data: positive exchange bias, magnetic properties of the top FM layer, and different $\mathrm{Ni}_{25} \mathrm{Mn}_{75}$ thickness. 